\title{
(a) Discovery in FHS
}

(i) Identify metabolites nominally associated $(p<0.05)$ with $\Delta \mathrm{BMI}$

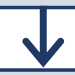

(ii) Perform stepwise regression and cross validation to build a multivariate model consisting of metabolites for predicting $\triangle \mathrm{BMI}$

(iii) Use metabolites and effect size estimates in stepwise model to calculate MRS

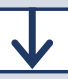

(iv) Test for association between $\triangle \mathrm{BMI}$ and MRS

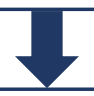

(b) Replication in MCDS

(i) Use metabolites and effect size estimates in FHS stepwise model to calculate MRS

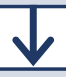

(ii) Test for association between $\triangle \mathrm{BMI}$ and MRS

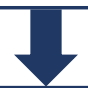

\section{(c) Association Analyses in FHS and MCDS}

(i) Investigate relationships between $\triangle \mathrm{BMI}, \mathrm{MRS}$, and other risk factors

(ii) Use logistic regression to predict future T2D status using MRS

(iii) Perform GWAS to identify genetic loci for MRS and MRS metabolites 